\title{
Prediction of hydraulic and acoustic properties of open-graded mixes
}

\author{
A.Umiliaco \& A. Benedetto \& F. Tosti \\ Roma Tre University, Rome, Italy \\ G. Di Mino \& G. Rodonò \\ University of Palermo, Palermo, Italy
}

\begin{abstract}
The use of open-graded mixes increases significantly the hydraulic permeability of pavement and contributes to reduce the noise impact in comparison to traditional pavement. Such characteristics are very appealing and would greatly increase driver's safety. However, during service life, the pores tend to be clogged by many factors among them secondary traffic compaction and filling of voids by fine materials are relevant, especially on heavy routes. In this case the noise reduction and drainage capabilities will gradually disappear, causing serious problems for road safety and maintenance. In this paper we propose a simulation approach in order to predict the drainage and acoustic behavior of open-graded mixes during service life. This numerical procedure allows to investigate the correlations between some indicators, extrapolated from the simulation, and the behavior of open-graded mixes, in order to optimize the design of the mix and the road maintenance.
\end{abstract}

\section{INTRODUCTION}

\subsection{Type area}

Open graded pavements are considered potentially to be a sustainable engineering solutions to increase the road safety, avoiding infiltration-related distress caused by the presence of water and reducing noise impact produced at the tire pavement contact. These asphalt mixes with high degrees of porosity (opengraded mixes) provide many advantages of their use than normal pavement materials presenting a high value of the index of the voids and interconnection among them, but provide also some disadvantages related to the cost and the maintenance of the pavement.

Advantages and disadvantages of porous asphalt have been well established in the literature (Younger et al., 1994); in the following chapter a summary description is presented.

\subsection{Advantages of HMA}

Reduction in splash and spray, reduced aquaplaning: compared to dense mixes, surface water can drain through porous asphalt due to the large amount of continuous pores in the structure. The material provides good visibility under rainy conditions, thus preventing the reduction in traffic flow volumes, which normally accompany rain. In addition, the ab- sorption of surface water is effective in reducing aquaplaning which occurs when vehicles move at high speeds on a thin water layer. It has been shown that porous asphalt contributes to the reduction of the number of accidents in rainy days (Maupin, 1976).

Reduction in light reflection and headlight glare: because porous asphalt acts as a drainage layer, enabling rainwater to percolate through the mix, thus light reflection and headlight glare, some of the dangerous factors for drivers especially in night time, decrease dramatically and lane markings are enhanced clearly on wet surfaces.

Improvement in Skid Resistance, Reduction in vehicle rolling resistance: increasing skid resistance under wet conditions is one of the main reasons for using porous asphalt. Assuming that a rougher wearing course would increase frictional properties. In Oregon friction properties of porous asphalt were compared with dense graded asphalt. The data accumulated indicated that porous asphalt mixes had slightly improved friction properties in dry conditions and a much improved friction properties during rainy conditions when free water was present on the pavement (Moore et al., 2001). Skid resistance is a function of macro and micro textures. At high speeds, the contribution of the macro texture is more dominant. In the A38 Burton trial section, 1987, Porous asphalt showed a skid resistance at least as 
good as that of the HRA (hot rolled asphalt) (Daines, 1992). In Japan, it is reported that the skid resistance of porous asphalt was initially the same as conventional nonporous asphalt, but this value increased gradually during the service life, whereas the dense mixes did not show any significant change. In addition, fresh porous asphalt layers may have a reduced skid resistance due to the bitumen film on the aggregates exposed to the surface. It is noteworthy to mention that some Swiss experts recommend not using porous asphalt with aggregate size in excess of $16 \mathrm{~mm}$ on wearing courses. According to their experience, the use of larger top size aggregates may provide less skid resistance on wet road surfaces.

Rut-resistance: in Japan, despite its high porosity, a number of trial sections show lower permanent deformation on porous asphalt than other dense mixes. Tighter aggregate skeleton in porous asphalt may contribute to withstand the load under traffic (EHRFJ, 1993). On the 1987 Burton trial in the UK, the deformation rate of this porous asphalt section in the near side lanes was less than $2 \mathrm{~mm} /$ year and 0.5 $\mathrm{mm} /$ year on average after 8 years trafficking. This result was evaluated as an acceptable rate in Britain. Although deformation of pavement depends on several conditions, such as climate, traffic intensity and loads, porous asphalt may provide acceptable rut resistance compared to other dense mixes (Daines, 1992).

Noise Reduction: road surfaces are laid with coarse macro-texture, which are in contact with the tire tread. This texture is known to contribute to the noise absorption between the surface and the tire. Many trial sections show lower noise levels on porous asphalt, which may be $6 \mathrm{~dB}(\mathrm{~A})$ lower than concrete layers (Tesoriere et al., 1989) or 2 to $6 \mathrm{~dB}(\mathrm{~A})$ lower than the HRA (Nicholls, 1996). According to the Swiss standards, under dry conditions in a 70 $\mathrm{dB}(\mathrm{A})$ area by using porous asphalt a noise reduction of $5 \mathrm{~dB}(\mathrm{~A})$ can be achieved (SN 640 433b, 2001). Swiss experience also indicates an advantage on the high speed traffic lanes in excess of about $80 \mathrm{~km} / \mathrm{h}$. Although the noise level of porous asphalt on the lower speed lanes is almost the same as other conventional dense mixes, porous asphalt is still effective in reducing the noise in the frequency range of 1.25 to $2 \mathrm{kHz}$ at $60 \mathrm{~km} / \mathrm{h}$ (Köster, 1991). The experience in the Netherlands indicates that on the lower traffic speed lanes less than $70 \mathrm{~km}$, the noise level of porous asphalt is even higher than dense mixes due to its rough macro-texture on the surface. To improve this aspect, 2 layer porous asphalt (twinlay) was developed (Bochove, 1996). It consists of a bottom layer of the porous asphalt with a coarse single grained aggregate $(11 / 16 \mathrm{~mm})$ and a thin top layer of fine graded porous asphalt $(4 / 8 \mathrm{~mm})$. This doublelayer structure can contribute to reduce the traffic noise at any vehicular speed. According to their report, additional advantages of the twinlay are better clog resistance against dirt and better cleaning properties. Therefore, this unique structure is expected to be introduced in their urban areas on a regular basis to meet the high environmental demand. Japanese experience reveals that porous asphalt is effective in noise reduction, but that this advantage is gradually lost over the years due to a decrease in mix porosity, especially in snowy areas where tire chains are used (JHPC, 1994). As an example from the USA in Oregon, two types of noise measurements were taken. The first was roadside noise and the second was interior vehicle noise. The results indicated that porous asphalt pavements reduce the noise in the higherfrequency zones. This conclusion is supported mostly from the roadside measurements and not from those taken in the interior of the vehicle, possibly since the higher frequencies are dampened by the vehicle

\subsection{Disadvantages of HMA}

Aging and Ravelling: although porous asphalt has many obvious advantages, there are also some disadvantages. One of the most critical factors in the performance of bituminous mixes is the tendency of the binder film on the surface of the aggregate to be continuously exposed to oxygen, sunlight, water etc. This results in binder hardening and a reduction in pavement service life (Hoban et al., 1985). When bitumen hardens, aggregates can be stripped easily from the asphalt mixes. It is well known that, due to its high porosity, porous asphalt ages much faster than conventional dense mixes. In full-scale road trials in the UK, the results conclude that the life of porous asphalt is ultimately limited by binder hardening with likely failure when its penetration drops below 15 pen (Daines, 1992). Another potential disadvantage of porous asphalt is the water sensitivity of the mix. Rainwater can penetrate through the porous matrix. Sometimes the water remains in the structure keeping the asphalt in wet condition for a long time. This moisture can cause some extra damage in porous asphalt by ravelling the binder film from the aggregate surfaces.

Reduction in Porosity: during service life, the pores tend to be clogged by dirt, dust or other clogging agents. On high speed lanes, tires produce a self-cleaning effect (Van Heystraeten et al., 1990). Thus clogging is more serious on low speed lanes or minor roads. With the loss of pores, the advantages of noise reduction and drainage function will gradually disappear. This is another serious problem for road maintenance. To overcome this disadvantage many types of cleaning methods, including vacuum vehicles with hydraulic jet water, have been developed to maintain the advantage of porous asphalt long term. However, no conclusion on the optimum type of cleaning method can be recommended. Porosity loss is also caused by secondary traffic compaction, especially on heavy routes. 
Shorter Service Life: due to the above listed disadvantages, the service life of porous asphalt surfaces is shorter than that of dense mix layers. In addition, it depends on several factors such as binder content and type, aggregate gradation, traffic volume and climate. Although previous experiences show an optimistic life expectancy of around 15 years, some maintenance should be necessary within about 5 to 8 years according to the results in many countries. Such maintenance costs for porous asphalt (from cleaning the clogged pores to replacement of those layers, which lost their drainage function) are considered higher than for the conventional asphalt. However, this does not mean that cost-effectiveness of porous asphalt surface is lower than that of other surface mixes. When this issue is discussed, the significant contribution of this pervious layer for social benefits, such as traffic safety and environmental issues, can not be ignored.

Winter Maintenance: snow and ice removal from porous surfaces requires at least twice the quantity of de-icing salt treatment compared to that of other dense mixes. However, the damage to porous asphalt due to salt is still unclear. Vehicular tire chains, spiked tires and snow ploughing sometimes cause severe damage on the open textured mixes requiring additional repair when the aggregates are stripped from the surface. Swiss standards recommend explicitly that porous asphalt not be used in areas where chains and spiked tires are used (SN 640 433b, 2001). CEN suggests an abrasion test by studded tires to evaluate the chain damage (EN13108-7, 2006).Japan also applies either a similar test for porous asphalt, which was originally developed for dense mixes in snowy areas, or a decrease in the temperature down to $-20{ }^{\circ} \mathrm{C}$ in the Cantabro test (Japan Highway Public Corporation, 1994). It should be noted that, because of the lower thermal conductivity of the porous asphalt, in winter this surface may colder than dense asphalt (Köster, 1991). Therefore, on the porous asphalt surface, snow tends to settle earlier and remain longer, also ice forms earlier when the roads are wet (Nicholls, 1996).

All these studies and the disadvantages of opengraded asphalt show that one of the most important factor that influence the acoustic and drainage properties of the HMA and their behavior during the service life is the design of the mix, that affects also its durability and its safety. The mix design appears in this case as an optimization problem. However, because there are no analytical nor empirical model available, it often happens that the mixture is designed adopting a minimum or overestimated standard for the hydraulic and acoustic characteristics of the mix. This oversize produces many disadvantages and problems from an economic and environmental point of view (Benedetto et al., 2007).

In this paper we propose a simulation approach in order to predict the drainage and acoustic behavior of open-graded mixes during service life. In detail we have generated, using a Random Sequential Adsorption model, synthetic HMA samples starting from the same grading and bitumen contents of real samples produced in laboratory by volumetric methods. For each sample we have considered three number of gyrations which measure the mixture compaction during paving $\left(\mathrm{N}_{\mathrm{des}}\right)$, during the operational phase $\left(\mathrm{N}_{\text {int }}\right)$ and at the end of the service life $\left(\mathrm{N}_{\max }\right)$. After the numerical generation of samples we have simulated the unsteady flow of water through Lattice-Boltzmann method inside the specimens for each step in order to evaluate the expected permeability and we have determined an absorption coefficient, using a stationary waves device applied on the real sample, to evaluate the acoustic properties.

This numerical procedure allows to investigate the correlations between some indicators, extrapolated from the simulation, and the behavior of opengraded mixes, in order to optimize the design of the mix and the road maintenance

\section{2 . SIMULATION MODEL}

\subsection{Numerical generation and indicators}

The simulation model for the numerical generation of asphalt sample is based on the characterization of the microscopic structure of the sample. In particular this model allows to define the parameters that identify the distribution and the position of the constituents (aggregates, bitumen and air voids) of the open graded mixes. In order to define these parameters the model uses the Random Sequential adsorption (RSA) for the numerical generation of the sample. RSA is a simple but fundamental problem in statistical physics. Objects are added randomly, one at a time, to a d-dimensional space. They must not overlap with previously added objects. In the present case (Benedetto, 2009) the space is two-dimensional, why simulating a cross section of the sample, or three-dimensional, why simulating fully the sample. As the process of adding objects is proceeding it becomes more and more difficult to find regions where the objects can find a new place. Theoretically when no further addition is possible the process is reaching the so called "jamming limit" (Meakin et al., 1992).

The single particle of aggregate is approximated here by a sphere. The diameter of the sphere is an approximation of the specimen size. Assuming that the asphalt production is a random process, the RSA can be accepted as a good approximation of the mixture forming. In general it is evident that the spherical approximation is not always very realistic for this purpose, but in the case of porous HMA mix this approximation has been validated at a first stage (Benedetto, 2009). Eventually the model could be upgraded considering also different shapes for 
the particles if needed. Similar examples are discussed in the literature (hyper-spheres, ellipses, rectangles, parallel squares) (Manciu et al., 2004). The case of spheres is well and diffusely discussed in the literature (Cooper, 1988) if the particles have the same diameter. The case of different sizes has been rarely investigated. However some applications and studies can be found in the literature about the binary (Talbot et al., 1989) and polydisperse mixtures (Tarjus et al., 1991).

Here the case of a polydisperse mixture, two phase (solid, air), is simulated according to the sizes distribution of the aggregate. The spheres have diameters from a minimum value $\delta_{\min }$ to a maximum value $\delta_{\max }$. The method is here applied to simulate real porous hot-mix asphalt samples,

with the dimension and the shape of a cylinder $\Phi 0.15$ $\mathrm{m}$ and $0.05 \mathrm{~m}$ high. The RSA algorithm selects one diameter (D) of a single sphere at random and one point $(\mathrm{x}, \mathrm{y}, \mathrm{z})$ within the three-dimensional domain, that is the position of the center of the sphere. The selection of the diameter is made within the range of possible diameters $\left(\delta_{\min } \leq \mathrm{D} \leq \delta_{\max }\right)$. The final set of selected diameters must accord to the distribution of aggregate sizes. At this scope the expected grading is divided in mutually exclusive but exhaustive classes (i.e. class $i$ is from diameter Di to diameter Di+1). Within each class a set of diameters is extracted at random in a way that the final distribution of particles all over the classes accords to the real grading of aggregates.

Each sphere inserted in the domain is labelled with a number that points to that sphere in a list where all the inserted spheres are associated to the coordinates of the position of the center and to the diameter. Basically it facilitates the process of checking for overlapping. In the tests we did not attempt to reach the jamming limit because the real samples are not compacted at the maximum theoretical limit. Otherwise the simulation is stopped when the rate between solid, as sum of the particles volumes, and the volume of voids tends to the real rate. Finally the distribution of spheres sizes is checked, to verify the consistency of the simulation respect to the real grading.

Two different approaches have been adopted. A complete three dimensional approach and a simplified twodimensional approach. A 2D sample is shown in Figure 1.

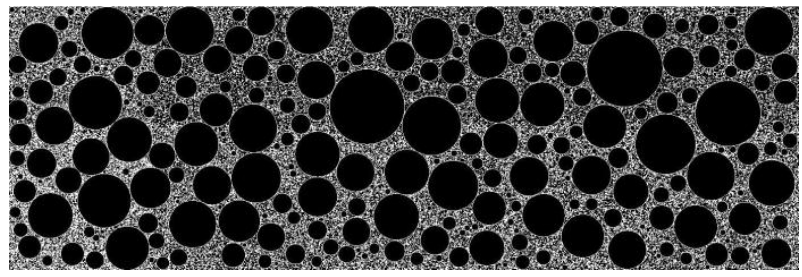

Figure 1. Example of two dimensional generation of asphalt sample.

It has been verified (Benedetto, 2009) that this second approach (2D) gives results that are strictly correlated to the three-dimensional approach and it can be considered absolutely more efficient, under a computational point of view, if a full and accurate generation of the real sample is not required.

According to one aggregate grading and one bitumen content it is possible to generate how many different samples how the different random seeds are. This casual procedure simulates the real procedure of sample making. It is well known that the laboratory procedure to make samples produces, from the same initial conditions, different results, also under rigorous standards and methods. This is the reason why, in the laboratory tests, a number of samples is required to calculate the average values of mechanical or hydraulic properties. For obvious reasons of time and cost this number of samples is always very limited (generally four samples). The numerical simulation makes it possible to generate a great amount of virtual samples. Over this great amount of samples it is possible to extract more stable and representative averages, following a Monte Carlo procedure (Von Neumann, 1951).

Once the set of samples have been simulated starting from the grading of aggregates and from the bitumen content, according to (Benedetto, 2009), it is possible to extract the average values, of some indicators representing geometrical and topological characteristics of the mixtures. The first step is the definition of these indicators .

Here 15 different indicators, according to (Benedetto, 2009), are examinated. These indicators have been selected considering the main factors that are expected to play significant role in the behavior of the mixture.

Table 1 lists all the 15 indicators.

Table 1. The indicators extracted from simulated samples

\begin{tabular}{|c|c|c|}
\hline $\mathrm{N}^{\circ}$ & Indicator & Definition \\
\hline 1 & $\mathrm{I}_{1}$ & Expected value of bitumen film thickness $z_{i}$ \\
\hline 2 & $\mathrm{I}_{2}$ & Number of contacts among all the particles \\
\hline 3 & $\mathrm{I}_{3}$ & Number of contacts among big particles \\
\hline 4 & $\mathrm{I}_{4}$ & Number of contacts among little particles \\
\hline 5 & $\mathrm{I}_{5}$ & $\begin{array}{l}\text { Number of contacts between big and little } \\
\text { particles }\end{array}$ \\
\hline 6 & $\mathrm{I}_{6}$ & Number of contacts Type I among particles \\
\hline 7 & $\mathrm{I}_{7}$ & Number of contacts Type II among particles \\
\hline 8 & $\mathrm{I}_{8}$ & Expected value of the distance among contact \\
\hline 9 & $\mathrm{I}_{9}$ & $\begin{array}{l}\text { Expected value of the distance among contacts } \\
\text { weighted by the sum of particles radii }\end{array}$ \\
\hline 10 & $\mathrm{I}_{10}$ & $\begin{array}{l}\text { Expected value of the distance among contacts } \\
\text { Type I }\end{array}$ \\
\hline 11 & $\mathrm{I}_{11}$ & $\begin{array}{l}\text { Expected value of the distance among contacts } \\
\text { Type II }\end{array}$ \\
\hline 12 & $\mathrm{I}_{12}$ & Number of interspace between particles \\
\hline 13 & $\mathrm{I}_{13}$ & $\begin{array}{l}\text { Expected value of the specific surface of par } \\
\text { ticles }\end{array}$ \\
\hline 14 & $\mathrm{I}_{14}$ & $\begin{array}{l}\text { Expected value of the specific surface of par } \\
\text { ticles and bitumen film }\end{array}$ \\
\hline 15 & $\mathrm{I}_{15}$ & $\begin{array}{l}\text { The rate between the number of contacts }\left(\mathrm{I}_{2}\right) \\
\text { and the number of total particles }\end{array}$ \\
\hline
\end{tabular}




\section{HYDRAULIC PROPERTIES OF HMA}

The hydraulic characteristics of HMA are determined by the use of another numerical model that simulate the flow of water inside the asphalt sample. It is based on Lattice - Boltzmann method (LB). The LB method is a class of computational fluid dynamics methods (CFD) for fluid simulation, it has evolved from the theory of Lattice Gas Automata (LGA) (Von Neumann, 1940). Among various techniques, the LB method is widely accepted. It has proven to be extremely efficient in the simulation of fluid flow through the complex geometries due to the facility of implementing boundary conditions and to the numerical stability in a wide variety of flow conditions. The LB method approximates the continuous Boltzmann equation by discretizing a physical space with lattice nodes and a velocity space by a set of microscopic velocity vectors (Kutay, 2006). The lattice nodes are uniformly spaced in order to represent the voids and the solids, while the discrete set of microscopic velocities is defined for propagation of fluid molecules (Fig. 2). For each nodes of the lattice the macroscopic flow properties, density and velocity, can be calculated.

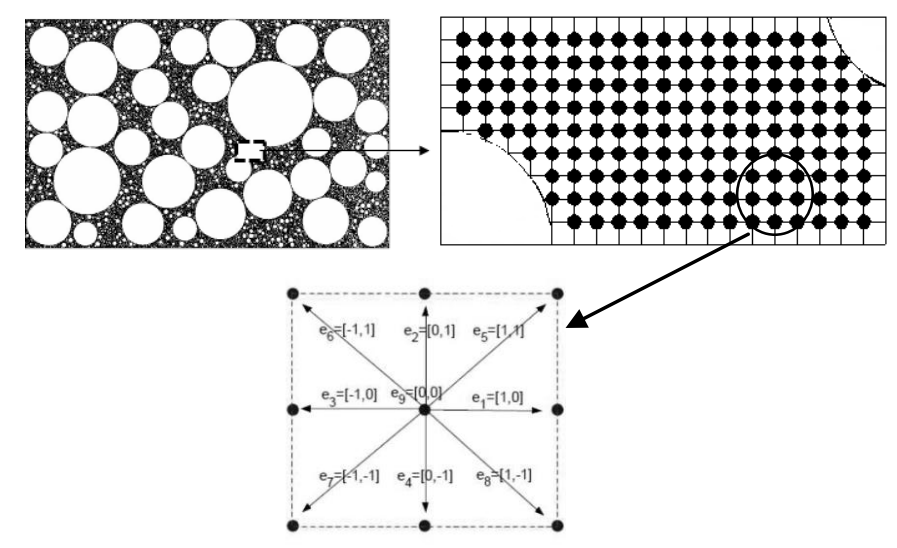

Figure 2. Lattice - Boltzmann Grid

The reliability of the model is already validated using experimental tests and theoretical calculation (Benedetto and Umiliaco, 2012). In particular starting from the generation of the synthetical sample and the subsequent simulation of the flow of water, as described previously, it is possible to determine a fundamental property related to the permeability: tortuosity. The tortuosity of a porous medium describes network complexity in porous media (flow paths). It depends on various parameters of the particles and settlement: the shape, size, and type of the grains, pores, and pore channels; mode of packing of the grains; grain size distribution; the orientation; and nonuniformity of the grains and it is defined as:

$$
T=\frac{L_{e}}{L}
$$

where $\mathrm{L}$ is the minimal geometrical distance between inlet and outlet and Le is an average effective hydraulic path length, of course it results Le > L.

The knowledge of this parameter through the simulation model, introduced in most of permeability models, allows to evaluate the permeability according to the following equation (Walsh and Brace, 1984):

$$
K=\frac{\gamma}{\mu} \frac{n_{e}^{3}}{b T^{2}} \frac{1}{S_{a}^{2}}
$$

where $\mathrm{K}$ is the hydraulic conductivity, Sa is the specific surface area, ne is the effective porosity, $\gamma$ is the unit weight of the fluid (water at $20^{\circ} \mathrm{C}$ ), and $\mu$ is fluid viscosity, $\mathrm{b}$ is a constant given as 2 for perfectly circular pore structure and 3 for rough texture on the pore surface.

In previous works the reliability of the results, obtained from the simulation and from the theoretical approach, were already validated by the comparison with some experimental outcomes, coming from laboratory tests using an hydraulic permeameter (Benedetto et al., 2007).

Figure 3 shows the correlation between the values of $\mathrm{K}$ measured and $\mathrm{K}$ predicted of two grading curves, from basaltic and silica - limestone aggregates, and three different percentage of bitumen for each of the four Marshall samples, for a total of 24 tests (Benedetto et al., 2007). The range of $K$ values accepted for open - graded asphalt pavements is $0,02-0,05 \mathrm{~cm} / \mathrm{s}$ and the range of $\mathrm{T}$ values accepted is $1,1-1,4$.

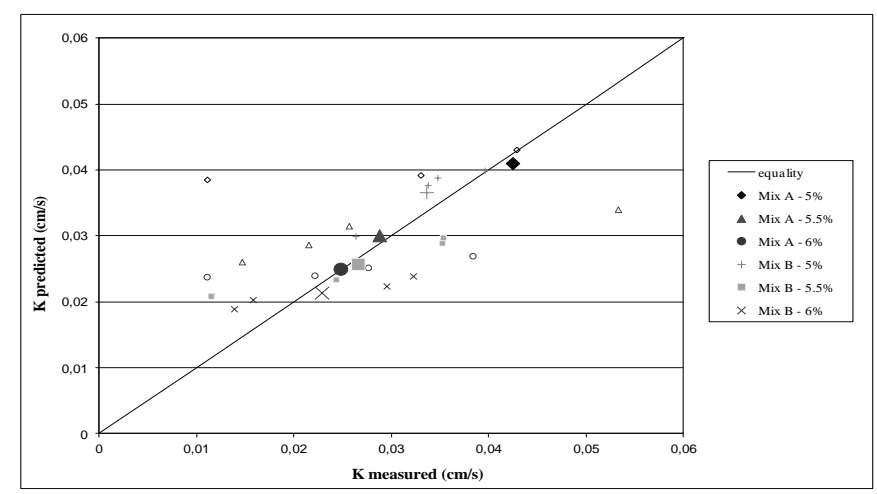

Figure 3. Validation of the model

\section{ACOUSTIC PROPERTIES OF HMA}

In order to assess the acoustic properties of HMA, as described previously, we manufactured samples by means of a gyratory compactor and we have considered three different steps, as mentioned, in terms of number of gyrations. They are consistent to the initial mixture compaction (Ndes $=30$ ), the compaction approximately after half of the service life (Nint $=70(\mathrm{G} 70, \mathrm{G} 75)$ or 50 (G80, G85) and the compaction at the end of the service life $(\mathrm{Nmax}=130)$.

In particular four grading curves have been selected, as shown in Figure 4. Two different percentage of modified bitumen have been used, 5.5\% for G70 and G75 and $5 \%$ for G80 and G85, to prepare three samples for each grading curve and number of gyrations, for a total of 36 samples.

For each of the 36 samples we have measured the acoustic characteristics by the determination of absorption coefficient $\alpha$, defined as the ratio between the inten 


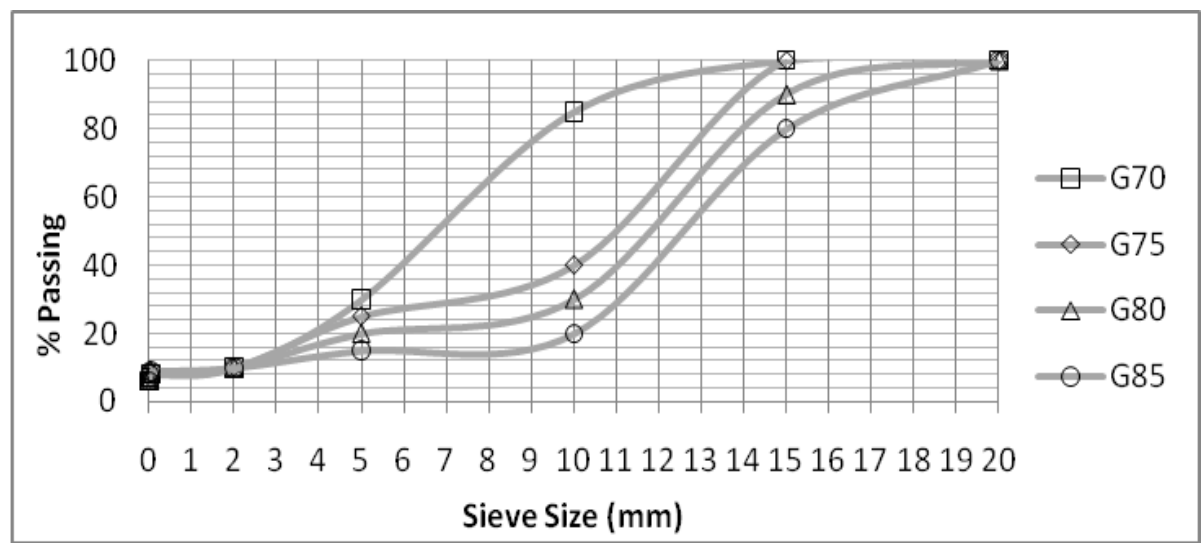

Figure 4. Selected grading curve.

sity of the absorbed acoustic wave and the intensity of the incident wave. This parameter is measured through a stationary waves device: Kundt's tube (Fig. 5). This tube has an internal diameter of $100 \mathrm{~mm}$.

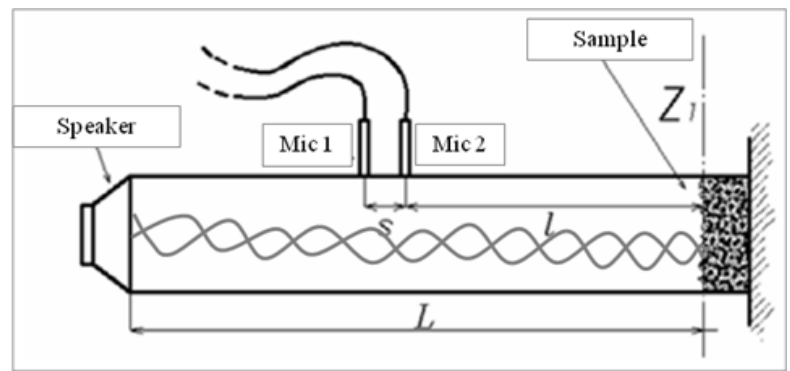

Figure 5. Stationary wave device - Kundt's Tube.

The measuring equipment consists of a computer for acquisition and data processing and a tube. At the two extreme parts of the tube there are respectively positioned: a speaker, that generates a tone of known frequency, and the sample (Fig. 5).

The signal generated from the speaker is a white noise, that has a frequency distribution almost constant in the range for the measurement $(200-600 \mathrm{~Hz})$.
The stationary waves propagate in the tube as plane waves which invest the sample and part of them are reflected. The overlap of the reflected waves and the incident waves return the typical phenomenon of stationary wave within the tube.

More in detail the absorption coefficient, as described previously, is obtained as a function of the chosenfrequency range, by determining a coefficient of reflection, obtained by measuring the sound pressure in two fixed locations (microphones) and by calculating, consequently, the response of the sample to the incident wave. The absorption coefficient is refer to the maximum value of the absorption spectrum of bands of narrow and large frequencies (one - third octave).

Figure 6 shows the measured absorption coefficient during the life cycle of the open-graded samples.

The plot position shows a good correlation between the absorption coefficient $\alpha$ and the voids content (\%), decreasing with the increase of the number of gyrations.

This correlation is showed in Figure 7.

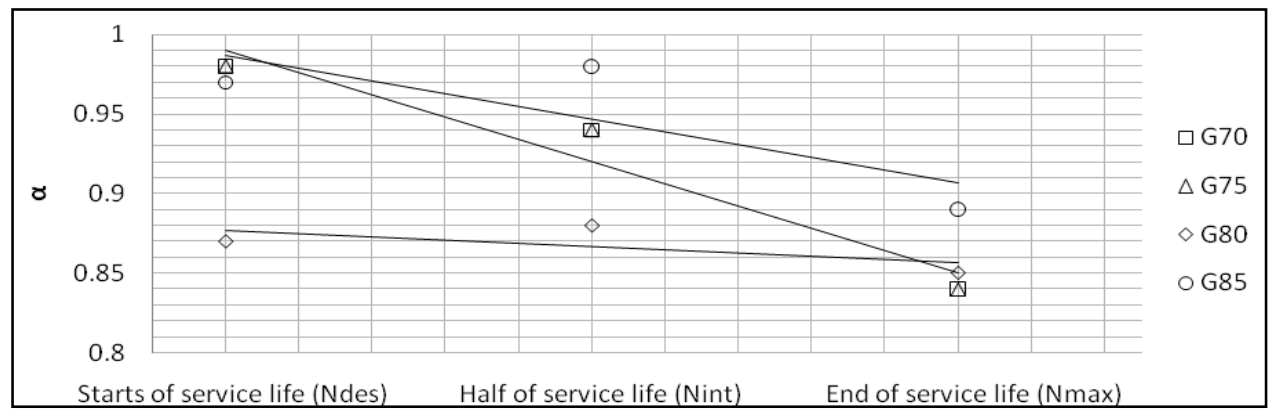

Figure 6. Absorption coefficient (a) versus service life.

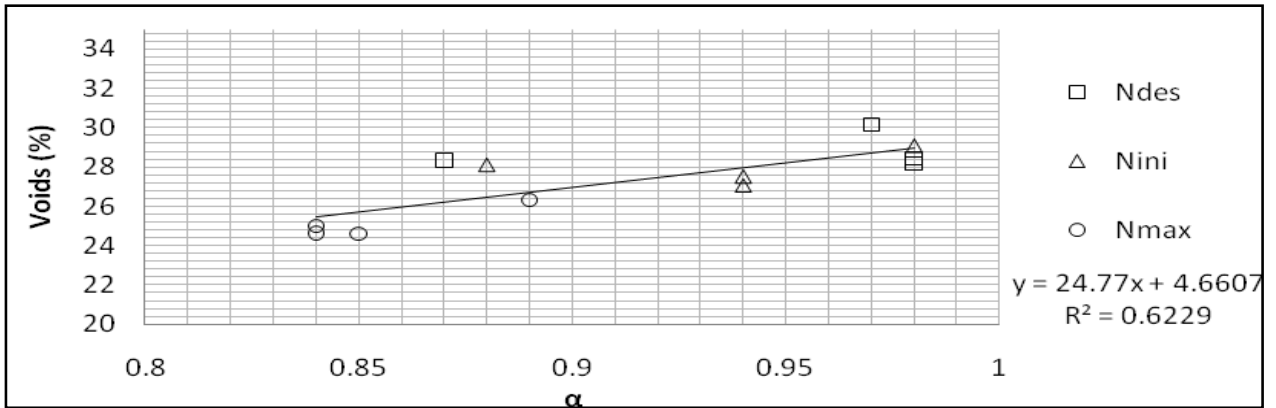

Figure 7. Absorption coefficient (a) versus void content. 


\section{CORRELATION}

In order to investigate a possible correlation between hydraulic permeability and the numerical indicators, starting from the grading curves (Fig. 4) we evaluate the drainage capability of the mixes coming from these grading curves using the Lattice - Boltzmann model, as described previously.

In particular for each of the synthetical samples the model simulates the different flow paths of the water, as show in Figure 8. The flow paths geometries allow to calculate the tortuosity (1) by tracking a band of most probable water paths and by assuming an average trajectory to measure the length of the paths.
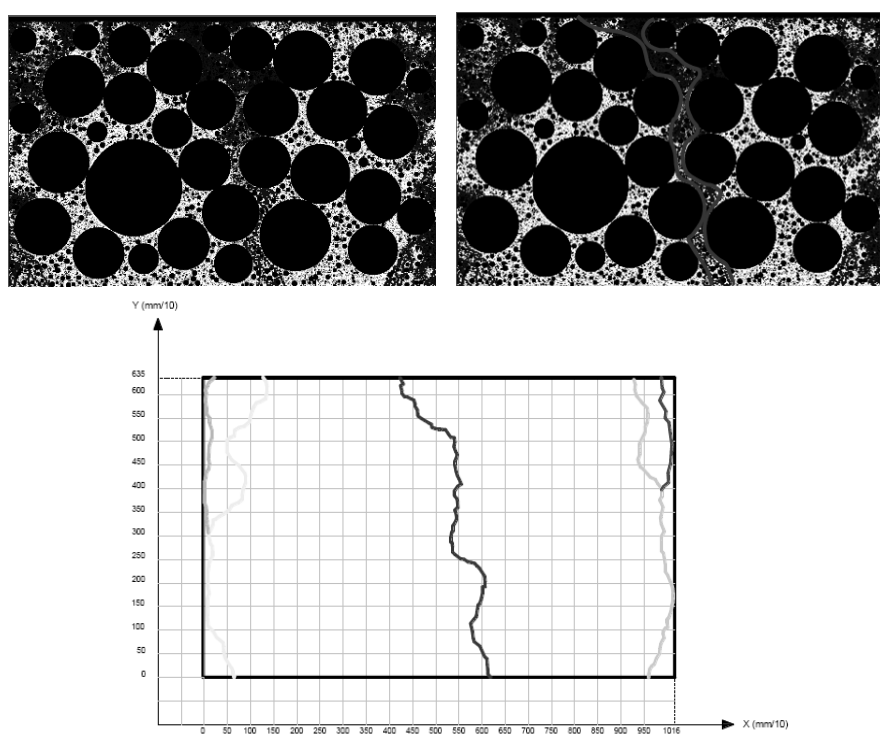

Figure 8. (a), (b), (c) identification and measurement of the length of water paths.
Using Walsh and Brace equation (2), where the values of parameters are evaluated from output of numerical generation, we are able to calculate the hydraulic permeability for each simulated samples.

The values of tortuosity and the related permeability for the virtual samples are shown in table 2 .

Table 2. Tortuosity and Permeability of the mixes

\begin{tabular}{clcl}
\hline Grading curve & Service Life & $\mathrm{T}$ & $\mathrm{K}(\mathrm{cm} / \mathrm{s})$ \\
\hline \multirow{3}{*}{ G70 } & Ndes & 1.41 & 0.0251 \\
& Nint & 1.43 & 0.0244 \\
& Nmax & 1.45 & 0.0237 \\
G75 & Ndes & 1.27 & 0.0309 \\
& Nint & 1.35 & 0.0273 \\
& Nmax & 1.40 & 0.0254 \\
G80 & Ndes & 1.18 & 0.0358 \\
& Nint & 1.27 & 0.0309 \\
& Nmax & 1.36 & 0.0269 \\
G85 & Ndes & 1.14 & 0.0383 \\
& Nint & 1.23 & 0.0329 \\
& Nmax & 1.26 & 0.0314 \\
\hline
\end{tabular}

Referring to the hydraulic properties, the in depth analysis of all the indicators shows a high correlation between the number of contacts per particle, extracted from the synthetic HMA sample (ratio between the number of contacts and the number of particles included in the reference domain, $\mathrm{I}_{15}$ ) and the values of tortuosity and permeability $(\mathrm{cm} / \mathrm{s})\left(\mathrm{R}^{2}=0.78\right)$ extracted from the synthetical samples, as the number of gyrations (Ndes - Nint Nmax) increases.

Figure 9 and Figure 10 show these correlations.

In Figure 11 the permeability values of the four mixes are compared.

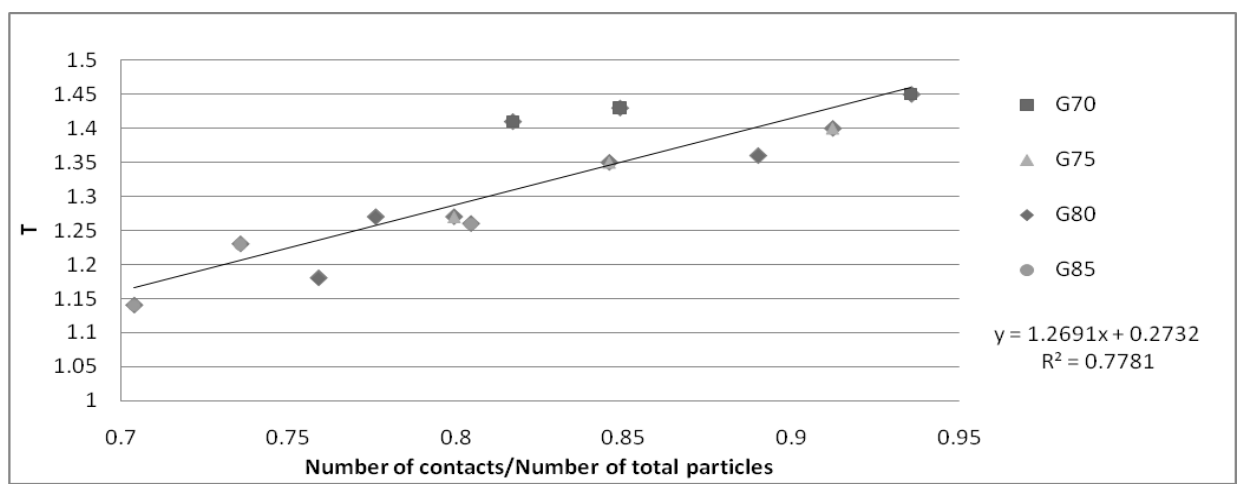

Figure 9: Ratio between number of contacts and number of particles versus tortuosity (T)

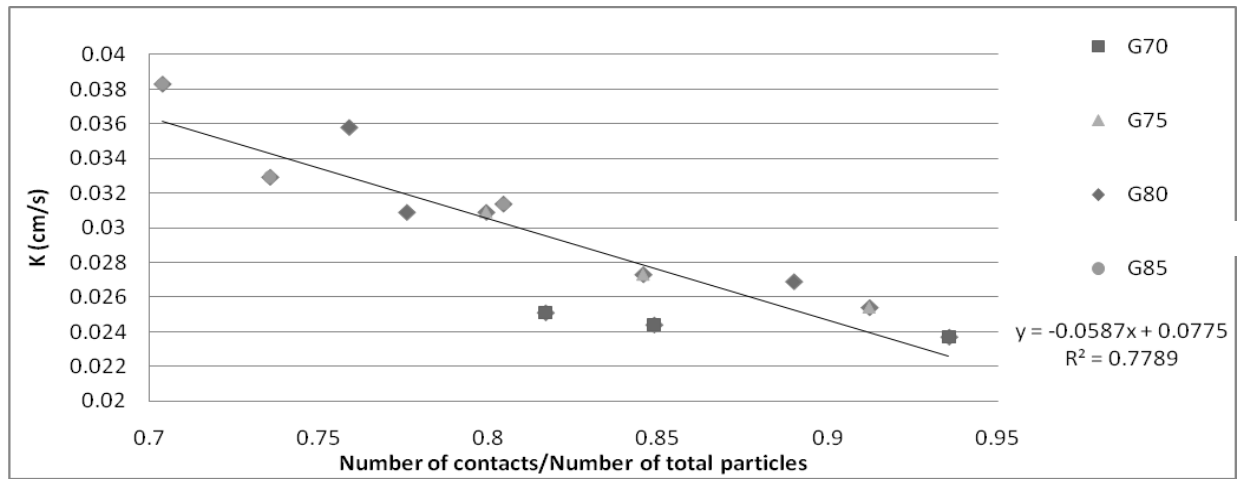

Figure 10: Ratio between number of contacts and number of particles versus permeability (K) 


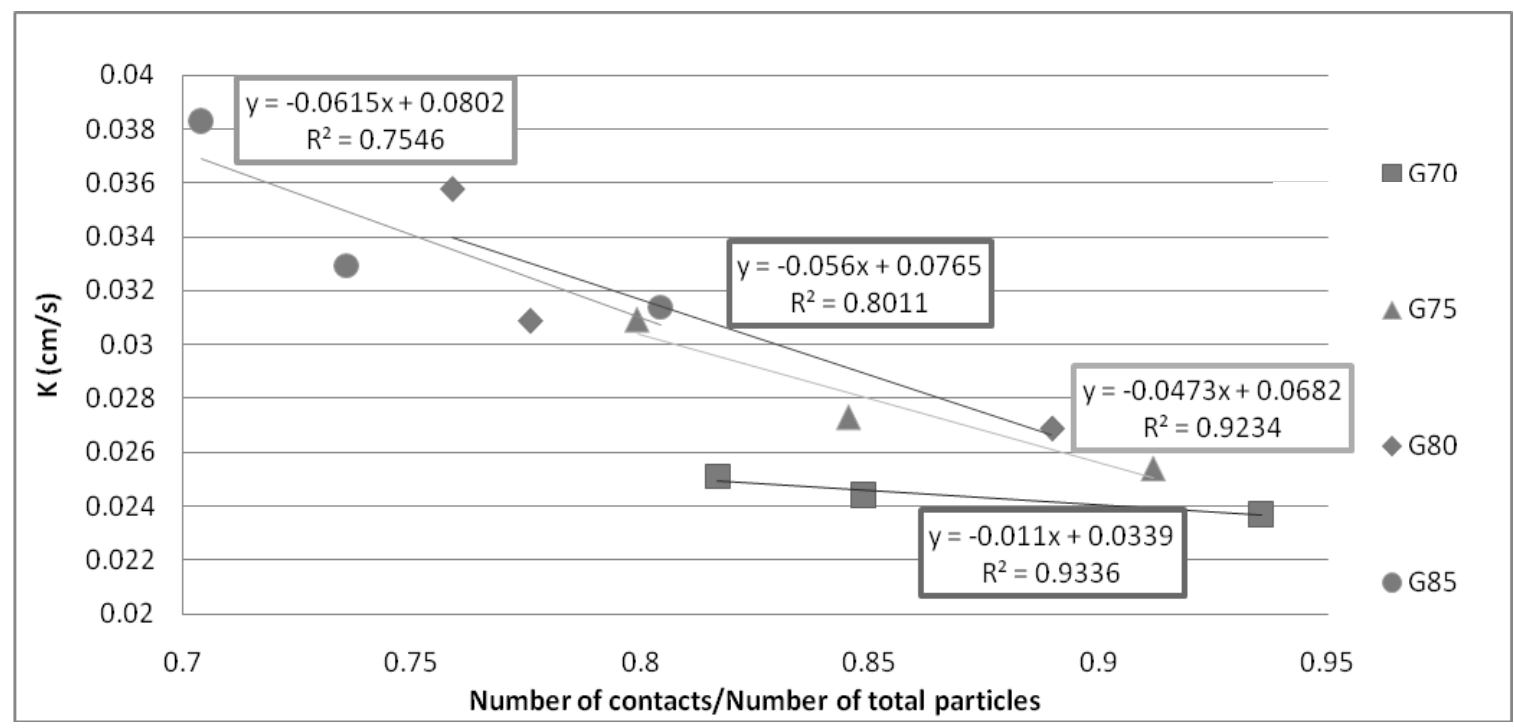

Figure 11: Ratio between number of contacts and number of particles versus permeability (K) for each grading curve.

Here the angular coefficients of the regression linear are mainly greater for coarser grading and lower for finer.

In detail Figure 11 shows that finer is the grading smoother is the decreasing trend of the permeability values while finer is the grading greater is the decreasing gradient for the permeability values, as the number of gyrations (Ndes - Nint - Nmax) increases.

It is possible to write in a general form the correlation equation between the permeability coefficient and the number of gyratory cycles, or life time, as it follows:

$$
K=K_{0}-\lambda t
$$

where:

$\mathrm{K}=$ permeability $(\mathrm{cm} / \mathrm{s})$;

$\lambda=$ coefficient of regression (function of the grain size distribution);

$\mathrm{t}=$ time related to the gyratory cycles and finally to the ratio between number of contacts and the number of inserted particles (Ndes - Nint - Nmax);

$\mathrm{K}_{0}=$ permeability at time $\mathrm{t}_{0}$ (function of the grain size distribution).

Referring to the acoustic properties, the in depth analysis of all the indicators shows a high correlation between the number of contacts per particle, as shown also for permeability, extracted from the synthetic HMA sample (ratio between the number of contacts and the number of particles included in the reference domain, $\mathrm{I}_{15}$ and the number of voids (\%) of the real samples, as the number of gyrations (Ndes - Nint - Nmax) increases.

This correlation is shown in Figure 12:

As expected, the figure shows a decreasing trend of the voids content as the number of gyrations increases. Accordingly, the increasing of gyrations cycles produces an increasing of the ratio between the number of contacts and the number of particles.

Moreover, if we consider the acoustic absorption coefficient $(\alpha)$, the correlation with the rate of contacts per particle is weaker (Fig. 13). This is reasonably due by the various levels of uncertainty as grain size characteris- tics, dimension and distribution of voids, that produces a not negligible dispersion in the plot position.

Under a quantitative point of view, figure 11 shows as the adsorption coefficient decreases as the voids content decreases.

It is clear that the absorption coefficient is reduced much more rapidly than the voids ratio, that decreases much slowly.

In a more complex and realistic model, where dimension and size distribution of particles are very variable, the absorption coefficient decreasing cannot be predicted easily, but an analogous trend is reasonably expected. More in depth it is expected that coarser is the grading smoother is the decreasing trend of the absorption coefficient, because the average final (after compaction) dimension of the voids is greater, while finer is the grading greater is the decreasing gradient for the absorption coefficient.

This assumption is qualitatively confirmed by the results, as it is shown in Figure 14, where the four materials, that have different grading curves, are compared. Here the angular coefficients of the regression linear are mainly greater for finer grading and lower for coarser.

It is possible to write in a general form the correlation equation between the absorption coefficient and the number of gyratory cycles, or life time, as it follows:

$$
\alpha=\omega t-\alpha_{0}
$$

where:

$\alpha=$ absorption coefficient;

$\omega=$ coefficient of regression (function of the grain size distribution);

$\mathrm{t}=$ time related to the gyratory cycles and finally to the ratio between number of contacts and the number of inserted particles (Ndes - Nint - Nmax);

$\alpha_{0}=$ absorption coefficient at time $t_{0}$ (function of the grain size distribution).

The coefficient of regression is expected to be a function of the particle size distribution, as well as the absorption coefficient at time $t_{0}$. 


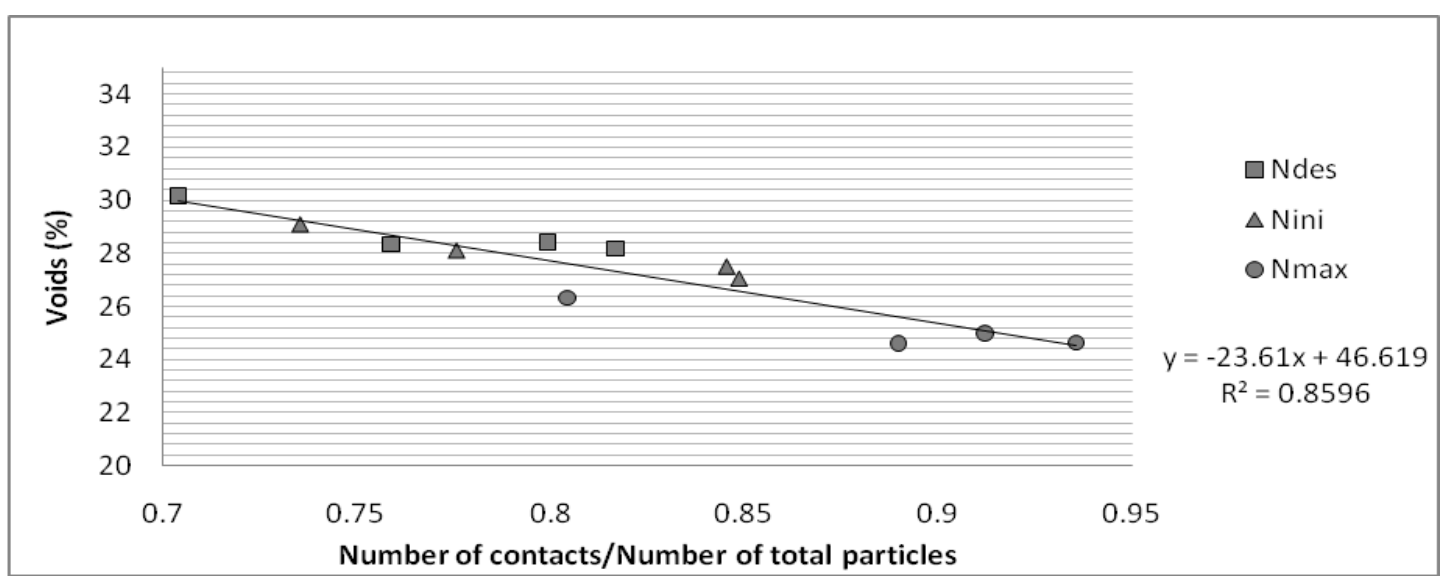

Figure 12: Ratio between number of contacts and number of particles versus voids content.

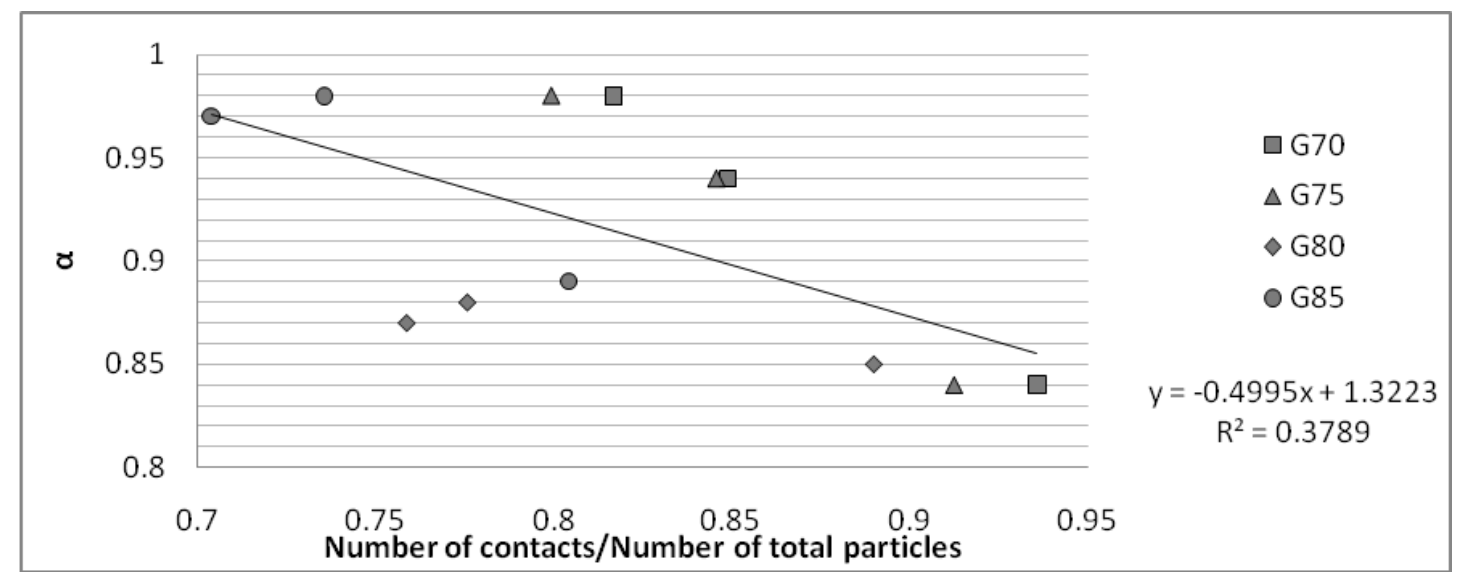

Figure 13: Ratio between number of contacts and number of particles versus absorption coefficient $(\alpha)$.

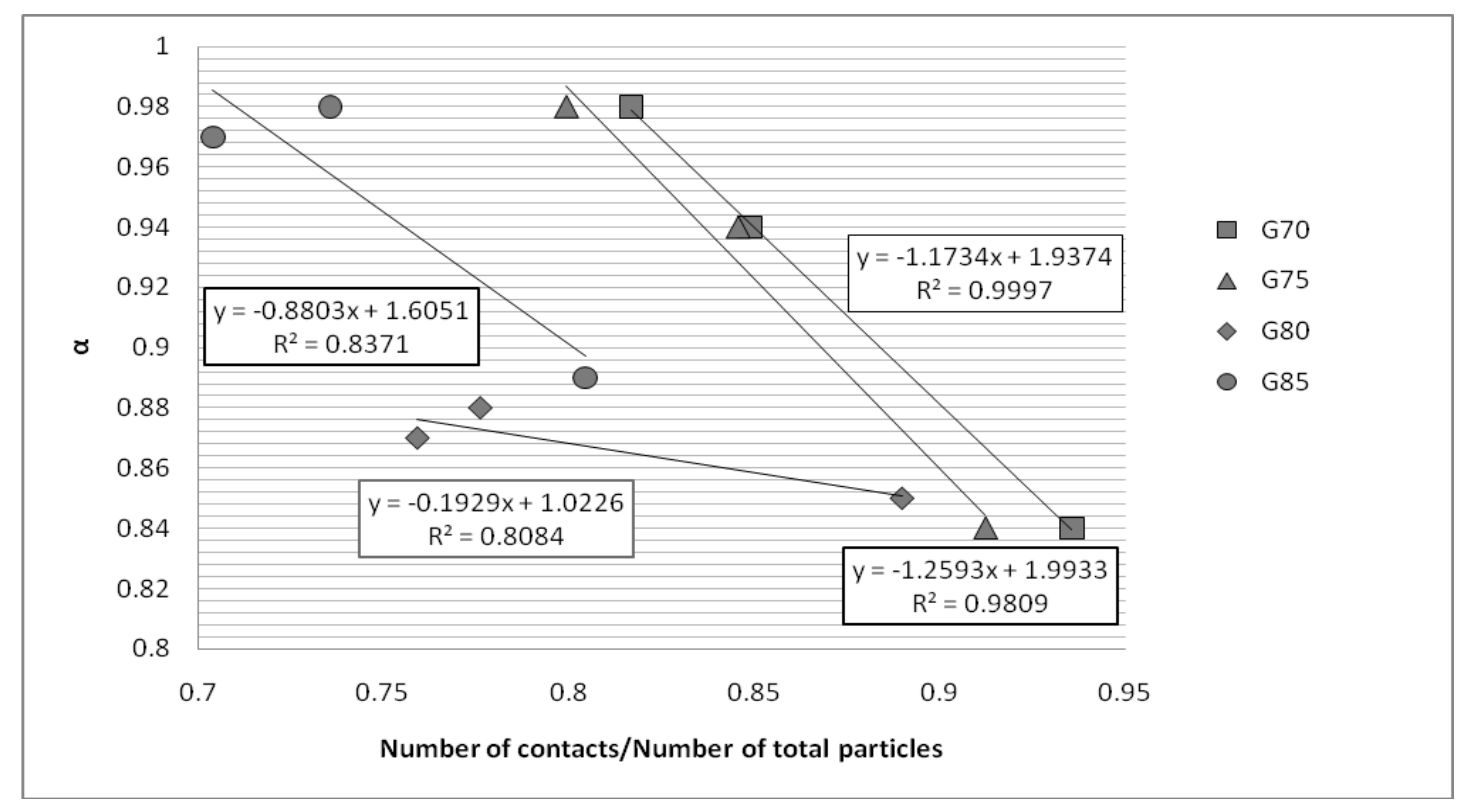

Figure 14: Ratio between number of contacts and number of particles versus $\alpha$ for each grading curve. 


\section{CONCLUSION}

Open-gradede pavements have many desiderable characteristics in order to increase road safety. They reduce noise, hydroplaning, splash and spray, rutting and cracking. However in order to improve upon this pavement type, it is necessary to correct some of the maintenance and construction problems. These problems can be reduce by a careful choice of the design of the mix in order to define and to optimize the most important properties (drainage and acoustic behavior) of HMA.

However it is very difficult to predict these features of the mixes, because it is a function of many variables related to the physical characteristics and to the microstructure (distribution, dimension and interconnection of voids) which make the evaluation very uncertain.

In this paper a numerical model is proposed to simulate open-graded sample in order to predict the hydraulic and acoustical features of HMA.

In particular number of contacts and dimension of voids seem to be the most significant indicators for predicting hydraulic (permeability) and acoustic properties (absorption coefficient) of HMA. More in depth the correlation between the permeability value and absorption coefficient with density of contacts appears as a function of grain size distribution and as a function of the increasing number of gyrations (Ndes - Nint - Nmax), that represents three different temporal steps of the lyfe cycle of the pavement.

It is possible to write in a general form the correlation equation between the permeability values and absorption coefficient with the life time (number of gyratory cycles). This correlation, that seems to be very promising, is very useful in order to optimize and to support the design of HMA to minimize costs and maintenance of the pavement. However additional experimental validations have to be carried out, in order to reach the needed reliability and a stable procedure.

\section{REFERENCES}

Benedetto, A. 2007. Open graded asphalt mixes: permeability and mechanical characteristics. International Journal of Pavement 6: 63-74.

Benedetto, A. 2009. Numerical simulation of mechanical and hydraulic characteristics of open graded mix. Proceedings of Conference on Maintenance and Rehabilitation of Pavements and Technical Control, Torino, Italy.

Benedetto, A., and Umiliaco, A. 2012. Evaluation of hydraulic permeability of open-graded asphalt mixes using a full numerical simulation. Journal of Materials in Civil Engineering, submitted.

Bochove, G.G. 1996. Twinlay, a New Concept for Porous Apshalt. Proc. Eurasphalt \& Eurobitume Congress.

Cooper, D. W. 1988. Random sequential packing simulations in 3D for spheres. Phys. Rev. A 38: 522-524.

Daines, M.E. 1992. Trials of porous asphalt and rolled asphalt on the A38 at Burton, Department of transport TRRL Report RR323, Transport and Road research Laboratory, Crowthorne.
EN13108-7. 2006. Provisional European Standard, Bituminous mixtures material specifications Part 7- porous asphalt (PA).

Express Highway Research Foundation of Japan. 1993. Report of Study and Research for Porous Asphalt. Tokyo.

Hoban, T. W. S., Liversedge, F., and Searby, R. 1985. Recent Developments in Pervious Macadam Surfaces. Proc. 3rd Eurobitumen Symp., The Hague, 635-640.

Japan Highway Public Corporation. 1994. Design and Execution Manual for Porous Asphalt. Tokyo.

Köster, H. 1991. Drainasphalt Beobachtungen des Verhaltens von hohlraumreichen Verschliessschichten unter Verkehr, Observation of the behavior of porous asphalt under traffic, VSS report Nr. 218.

Kutay, M. E., Aydilek, A. H., and Masad, E. 2006. Laboratory validation of lattice Boltzmann method for modeling porescale flow in granular materials. Computers and Geotechnics 33:, 381-395.

Manciu, M., and Ruckenstein, E.. 2004. Estimation of the available surface and the jamming coverage in the Random Sequential Adsorption of a binary mixture of disks. Colloids and Surfaces A: Physicochemical and Engineering Aspects 232: 1-10.

Maupin G W. 1976. Virginia's Experience with Open-Graded Surface Mix, Transportation research Record 595: 48-51.

Meakin, P., and Jullien, R.. 1992. RSA of disks of different sizes. Physical Review 46 (4): 2029- 2038.

Moore, L.M., Hicks, R.G., and Rogge, D.F. 2001. Design, Construction, and Maintenance Guidelines for Porous Asphalt Pavements. Transportation Research Record 1778.

Nicholls J.C. 1996. Review of UK porous asphalt trials. TRL Report 264, Transport Research Laboratory, Crowthorne, 1996.

SN 640 433b. 2001. Drainasphaltschichten, Konzeption Anforderung, Ausführung. Swiss, Standards for porous asphalt Original in German and French.

Talbot, J., and Schaaf, P. 1989. Random sequential adsorption of mixtures. Phys. Rev. A. 40 (1): 422-427.

Tarjus, G., and J. Talbot. Random sequential adsorption of polydisperse mixtures: asymptotic kinetics and structure. J. Phys. A., No. 24, 1991, pp. 913-917.

Tesoriere, G., Canale, S., and Ventura, F. 1989. Analysis of Draining Pavements from a Point of View of PhonoAbsorption. Proc. 4th Europian Symp., Madrid, 878-881.

Von Neumann, J. 1940. The estimation of probable error from successive differences. Aberdeen Proving Ground, Report 175,19 .

Von Neumann, J. 1951. Various techniques used in connection with random digits. US Nat. Bur. Stand. Appl. Math. Ser. 12: $36-38$.

Van Heystraeten, G., and Moraux, C. 1990. Ten Years' Experience of porous Asphalt in Belgium. Transportation Research Record 1265: 34-40, 1990.

Walsh, J.B., and Brace, W.F. 1984. The Effect of Pressure in Porosity and the Transport Properties of Rock. Journal of Geophysical Research 89: 9425-9431.

Younger, K., Partl, M., and Hicks, R.G. 1994. Evaluation of porous pavements for road surfaces. Interim Report, Oregon State University, department of civil engineering, Corvallis. 